\title{
Article \\ Multiple-Cycle Polymeric Extracellular Vesicle Precipitation and Its Evaluation by Targeted Mass Spectrometry
}

\author{
Jisook Park ${ }^{1}$, Eun-Bi Go ${ }^{2}$, Ji Sun Oh ${ }^{2}$, Jong Kyun Lee ${ }^{3}$ and Soo-Youn Lee ${ }^{4,5,6, *(D)}$ \\ 1 Samsung Biomedical Research Institute, Samsung Medical Center, Sungkyunkwan University School of \\ Medicine, Seoul 06351, Korea; js944837@skku.edu \\ 2 Samsung Biomedical Research Institute, Samsung Medical Center, Seoul 06351, Korea; \\ geb926@naver.com (E.-B.G.); jisun.oh@daum.net (J.S.O.) \\ 3 Division of Gastroenterology, Department of Medicine, Samsung Medical Center, \\ Sungkyunkwan University School of Medicine, Seoul 06351, Korea; jongk.lee@samsung.com \\ 4 Department of Laboratory Medicine and Genetics, Samsung Medical Center, \\ Sungkyunkwan University School of Medicine, Seoul 06351, Korea \\ 5 Department of Clinical Pharmacology and Therapeutics, Samsung Medical Center, Seoul 06351, Korea \\ 6 Department of Health Science and Technology, Samsung Advanced Institute of Health Science and \\ Technology, Sungkyunkwan University, Seoul 06351, Korea \\ * Correspondence: suddenbz@skku.edu
}

check for updates

Citation: Park, J.; Go, E.-B.; Oh, J.S.; Lee, J.K.; Lee, S.-Y. Multiple-Cycle Polymeric Extracellular Vesicle Precipitation and Its Evaluation by Targeted Mass Spectrometry. Int. J. Mol. Sci. 2021, 22, 4311. https:// doi.org/10.3390/ijms22094311

Academic Editor: Abdelnaby Khalyfa

Received: 15 March 2021

Accepted: 17 April 2021

Published: 21 April 2021

Publisher's Note: MDPI stays neutral with regard to jurisdictional claims in published maps and institutional affiliations.

Copyright: (c) 2021 by the authors. Licensee MDPI, Basel, Switzerland. This article is an open access article distributed under the terms and conditions of the Creative Commons Attribution (CC BY) license (https:// creativecommons.org/licenses/by/ $4.0 /)$.

\begin{abstract}
The multiple roles of extracellular vesicles (EVs) in pathogenesis have received much attention, as they are valuable as diagnostic and prognostic biomarkers. We employed polymeric EV precipitation to isolate EVs from clinical specimens to overcome the limitations of ultracentrifugation (UC), such as low protein yields, a large volume of specimens used, and time requirements. Multiplecycle polymeric EV precipitation was applied to enhance the purity of the EV fractions with a small sample volume. Then, the purity was assessed using a multiple reaction monitoring (MRM) panel consisting of alpha-2-macroglobulin (A2M), thrombospondin 1 (THBS 1), galectin 3 binding protein (LGALS3BP), and serum albumin (ALB). For purity evaluation, the EV fractions isolated from blood specimens were subjected to shotgun proteomics and MRM-based targeted proteomics analyses. We demonstrate that the modified method is an easy and convenient method compared with UC. In the shotgun proteomics analysis, the proteome profile of EV fraction contains $89 \%$ EV-related proteins by matching with the EVpedia database. In conclusion, we suggest that multiple-cycle polymeric $\mathrm{EV}$ precipitation is not only a more effective method for EV isolation for further proteomics-based experiments, but may also be useful for further clinical applications due to the higher EV yield and low sample requirements.
\end{abstract}

Keywords: extracellular vesicle; polymeric precipitation; multiple reaction monitoring; pancreatic cancer

\section{Introduction}

Extracellular vesicles (EVs) are spherical cell-derived membrane particles classified into exosomes (30-120 nm), microvesicles (100-1000 nm), and apoptotic bodies (800$5000 \mathrm{~nm}$ ) according to cellular origin, function, and biogenesis [1,2]. EVs contain bioactive molecules such as DNA, mRNA, microRNA(miRNA), proteins, and lipids, which are released by most cell types into body fluids (e.g., serum, urine, and cerebrospinal fluids) [3].

EVs are intracellular hormone-like messengers such as protein carriers or RNA carriers that are reported to be deeply involved in diseases and biological processes such as immune response regulation, blood coagulation, cell migration, cell differentiation, and cell-to-cell communication [4-8]. In particular, these vesicles are highly released in diseases including cancer and play a role in spreading proteins that cause disease to other cells [9]. Therefore, they have attracted much attention due to their roles under physiological and pathological conditions as diagnostic biomarkers and therapeutic targets $[10,11]$. In particular, the 
EV proteome profile has been highlighted as it is expected to be useful for diagnostic biomarkers and therapeutic targets [12-16].

Differential ultracentrifugation (UC) is the most traditional protocol used to isolate EV from various body fluids to date [17]. However, there are several limitations to its clinical application including the low yield of EVs, large volume of specimens, and above all, its time-consuming process. Polymer-based precipitation is more advantageous for clinical applications than UC because of the small sample volume, easy protocols, and short turnaround time $[18,19]$. However, contamination of non-EV proteins has been an obstacle to increasing the contents of EV proteins in polymeric methods [20]. The EVs acquired from biological fluids inevitably include high levels of serum-derived proteins, which are a major impediment to EV proteomics. UC is still considered the gold standard for EV isolation, especially for proteomics experiments.

Recent studies showed that multiple cycles of UC provided higher purity of EVs than classical UC purification [21]. Unfortunately, with multiple-cycle UC, a large sample volume is needed for EV isolation, whereas a smaller amount of EVs is recovered. Therefore, it is an urgent requirement to improve EV purification technology for further clinical applications.

A large sample size, poor yield of EVs, and the time-consuming process are major problems in the clinical application of EV biomarkers. The evaluation of impurities from the EV purification process is critical for downstream EV proteomics analysis. Evaluation of purity is limited to measurement of serum albumin using Western blotting without EVspecific proteins [22]. Development of an analysis method for purity evaluation is necessary. For quantitative analysis of newly discovered proteins, multiple reaction monitoring (MRM) is a more useful technique than other antibody-based methodologies (e.g., Western blotting or enzyme-linked immunosorbent assay) due to its rapid development and clinical application.

Polymeric reagents reduce the solubility of EVs and allow less-soluble components to precipitate out of the solution. This method produces a high yield of EVs, but contamination of non-EVs proteins is a major problem. We expected that repeated treatment of polymeric reagents can wash away contaminated proteins and increase purity. Therefore, we constructed a modified method that includes multi-cycle processing to enhance the purity.

Here, we present multiple-cycle polymeric EV precipitation with high purity, which may be applicable in clinical practice and provides EV marker proteins for purity evaluation. This is the first report on the usefulness of multi-cycle polymer-based reagent treatment compared with multi-cycle UC [23], including quantitative results of each EV fraction acquired from a different reagent processing cycle and verified by MRM assay.

\section{Results and Discussion}

\subsection{The Size Distribution and Morphology Analysis of EVs}

The EVs isolated from human serum by UC and multiple-cycle polymeric EV precipitation were characterized by TEM and a Zetasizer Nano ZS. The TEM and Zetasizer analyses were applied to confirm the size distribution and morphology of the isolated EVs (Figure 1). The average sizes of the UC and four-cycle EV fractions were $114 \mathrm{~nm}$ $(50-160 \mathrm{~nm})$ and $40 \mathrm{~nm}(25-80 \mathrm{~nm})$, respectively. The four-cycle EV fractions were a little smaller than the UC fractions. 
A

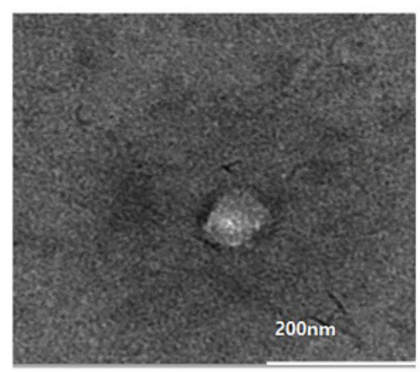

B

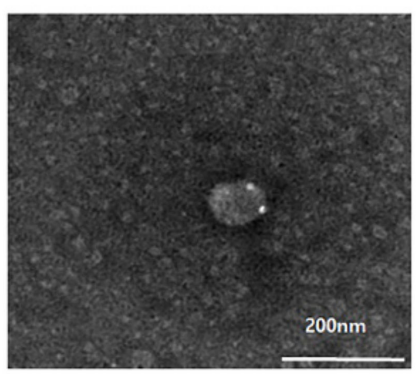

C

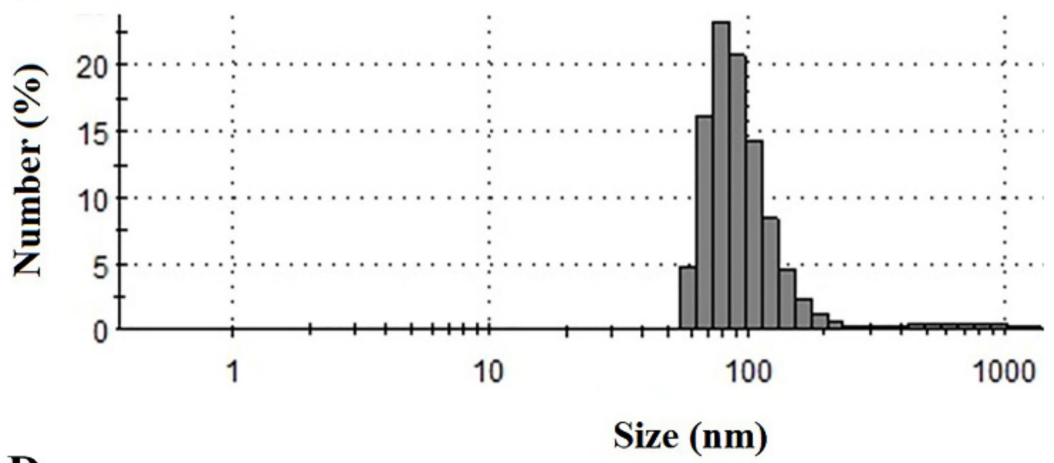

D

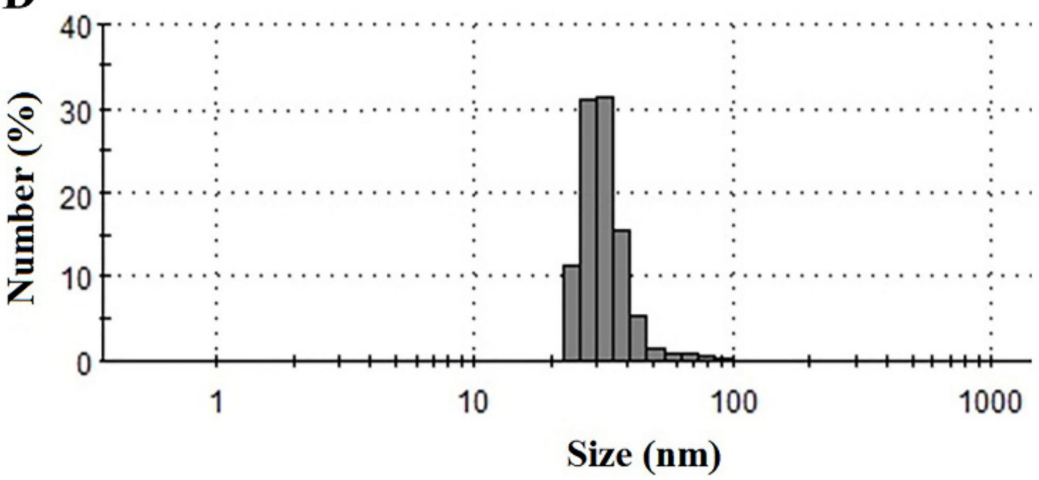

Figure 1. Characterization of EV fractions obtained from UC and multiple-cycle polymeric EV precipitation. Transmission electron microscopy (TEM) and dynamic light scattering (DLS) analyses were performed to determine the morphology of vesicles and particle size distribution in the $(\mathbf{A}, \mathbf{C}) \mathrm{UC}-\mathrm{EV}$ and $(\mathbf{B}, \mathbf{D}) 4$-cycle EV fractions.

\subsection{Identification of EV Proteins Using Proteomic Analysis}

A total of 612 proteins (unique peptides $\geq 2$ ) was identified in the four-cycle EV fractions from human serum by shotgun proteomic analysis. Of them, 539 proteins (89\%) were included in the EVpedia depository (http: / / evpedia.info, accessed on 18 May 2019), a public database for extracellular vesicle research. These proteins were highly enriched in extracellular exosomes, extracellular vesicles, and extracellular regions by functional enrichment analysis using FunRich version 3.1.3 (Figure 2). We could not prepare enough EVs fractions from UC experiments due to the limitations due to sample volume and very low recovery rate. Therefore, our current data were compared with the proteome profile from another investigation using multiple-cycle UC [21]. In brief, Kim et al. reported that 478 proteins including a single unique peptide were identified in LC-MS/MS analysis of EV proteins enriched from $4 \mathrm{~mL}$ of serum using five cycles of UC. Among these proteins, we extracted 273 having two or more unique peptides to compare with our identified proteins.

A total of 139 proteins was commonly identified in both the UC fraction and our four-cycle EV fraction, and 465 proteins were exclusively included in the four-cycle EV fraction. More plasma membrane proteins were enriched in the four-cycle EV fraction (99 proteins) than in the UC fraction (38 proteins) (Supplementary Table S1), and 27 clusters of differentiation (CD) proteins were identified in both fractions (Table 1). As a result, our data indicate that EV isolation by our protocol can be applied to various proteome-based investigations. 
A

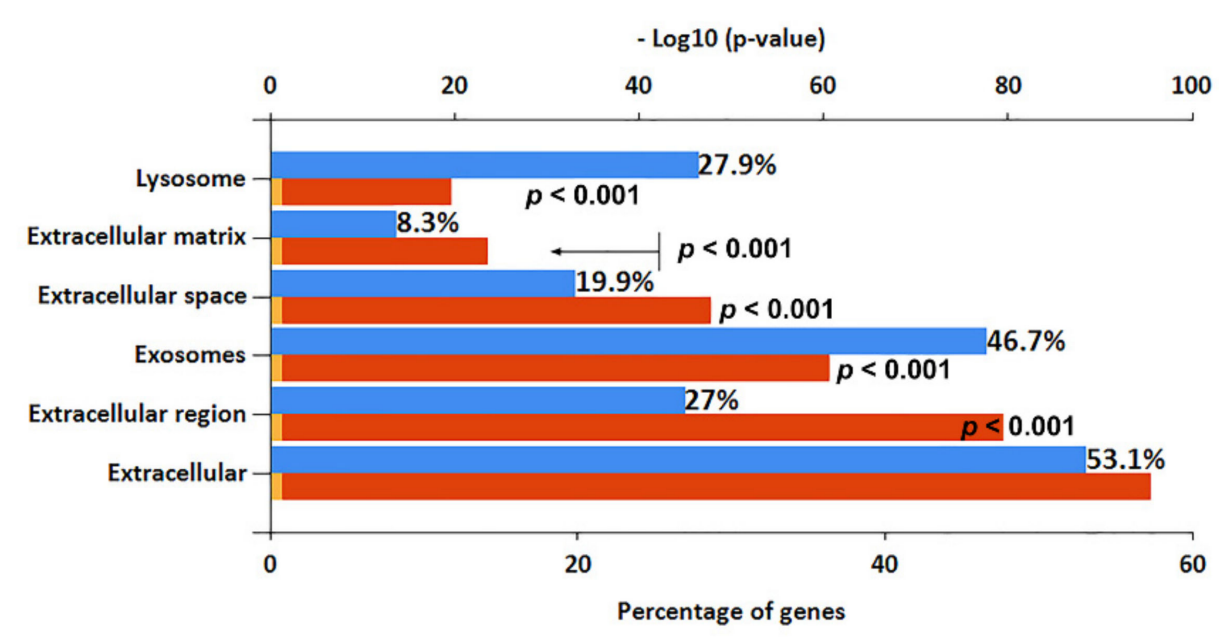

B

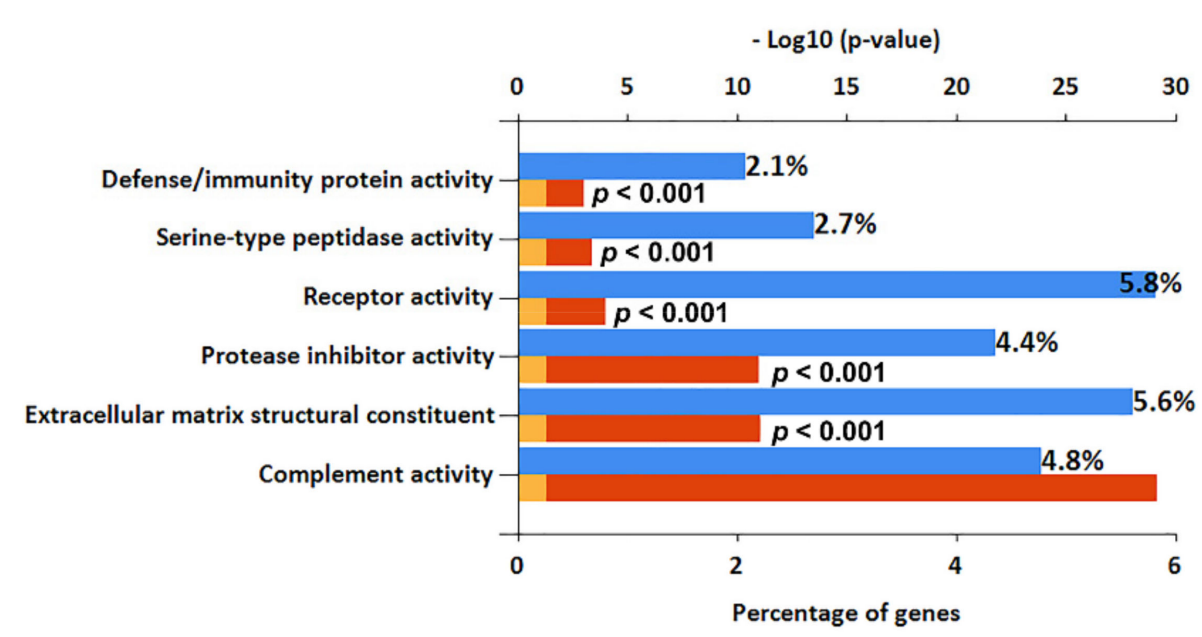

C

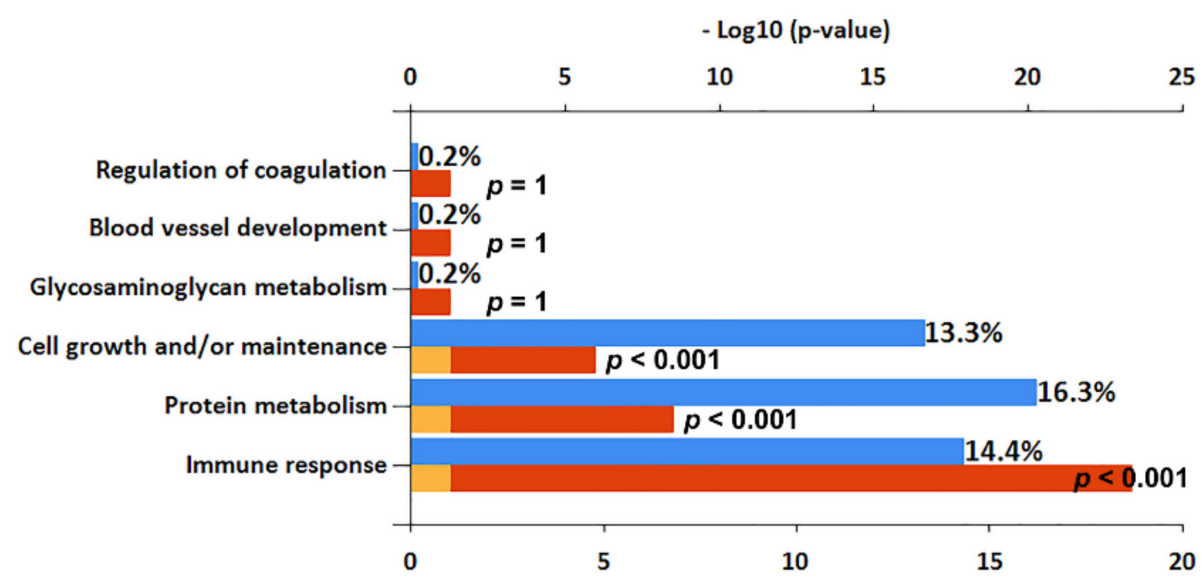

Figure 2. Functional analysis of identified proteins in 4-cycle EV fractions. Functional enrichment analysis was conducted using FunRich software: (A) cellular components, (B) molecular functions, and (C) biological process. 
Table 1. The 27 CD markers identified in the 4-cycle EV fraction.

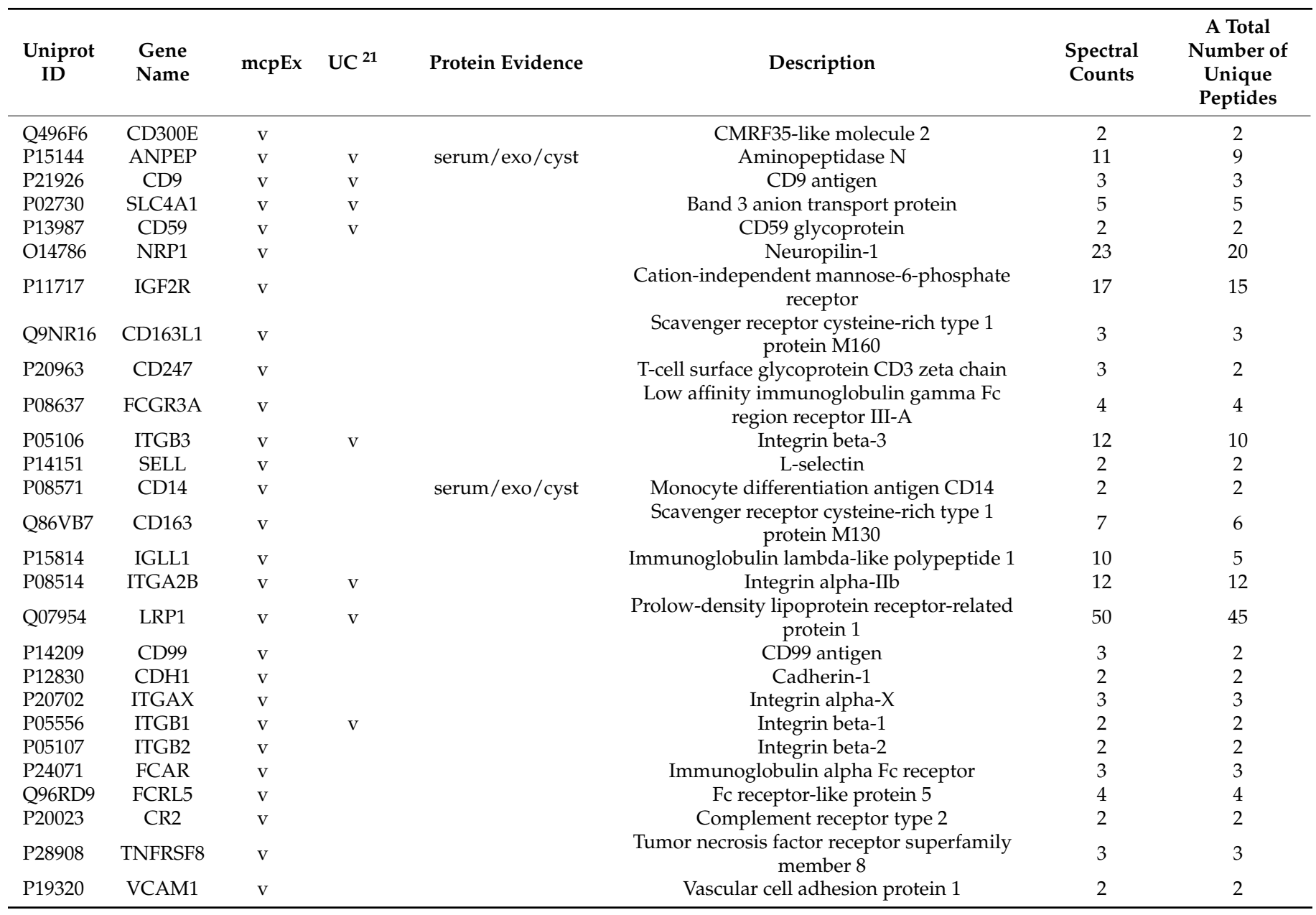

\subsection{EV MRM Assay Development}

A total of 234 MRM transitions were generated against the 26 proteins and 78 peptides common among the shotgun data and top $100 \mathrm{EV}$ proteins obtained using Skyline software. The MRM methods were experimentally refined with the EV fractions obtained from pooled serum. Of them, MRM assays of six proteins were finally established (Supplementary Table S2 and Supplementary Figure S1). We measured the levels of these proteins in the EV fractions (one to four cycles) obtained from pooled sera, of which three proteins, A2M, THBS1, and LGALS3BP, increased significantly after EV purification (Supplementary Figure S2). Therefore, these three proteins were chosen as EV marker proteins for the EV purity evaluation (Table 2).

\subsection{Purity Evaluation by Immunoblotting and MRM Assay}

Western blot analysis of CD9, CD63, and CD81 was performed to assess the losses and concentration efficiency of EV purification. In the current study, there was no significant intensity difference in the band of each protein between UC and 1-4-cycle EV fractions when an equal amount of the EV fraction was applied (Figure 3A). Meanwhile, its total protein amount was at least 4.6-fold higher than that of UC, even though the volume of the sample we treated was 16 times smaller (data not shown). 
Table 2. MRM-MS of extracellular vesicles along with representative peptide signatures used to assess isolated EV purity.

\begin{tabular}{|c|c|c|c|c|c|c|c|}
\hline \multirow{2}{*}{ HGNC Symbol } & \multirow{2}{*}{ Peptide Sequence } & \multirow{2}{*}{ Target Ion } & \multicolumn{2}{|c|}{ Intact $(\mathrm{m} / \mathrm{z})$} & \multicolumn{2}{|c|}{ IS (m/z) } & \multirow{2}{*}{$\mathrm{CE}(\mathrm{eV})$} \\
\hline & & & Q1 & Q3 & Q1 & Q3 & \\
\hline \multirow{3}{*}{ ABL } & \multirow{3}{*}{ FQNALLVR * } & $+2 \mathrm{y} 6$ & 480.8 & 685.4 & 485.8 & 695.4 & 26.2 \\
\hline & & $+2 \mathrm{y} 5$ & 480.8 & 571.4 & 485.8 & 581.4 & 26.2 \\
\hline & & $+2 \mathrm{y} 4$ & 480.8 & 500.4 & 485.8 & 510.4 & 26.2 \\
\hline \multirow{3}{*}{ THBS1 } & \multirow{3}{*}{ SITLFVQEDR * } & $+2 \mathrm{y} 8$ & 604.3 & 1007.5 & 609.3 & 1017.5 & 30.6 \\
\hline & & $+2 \mathrm{y} 6$ & 604.3 & 793.4 & 609.3 & 803.4 & 30.6 \\
\hline & & $+2 \mathrm{y} 5$ & 604.3 & 646.3 & 609.3 & 656.3 & 30.6 \\
\hline \multirow{3}{*}{$\mathrm{A} 2 \mathrm{M}$} & \multirow{3}{*}{ NEDSLVFVQTDK * } & $+2 \mathrm{y} 10$ & 697.8 & 1151.6 & 701.9 & 1159.6 & 34.0 \\
\hline & & $+2 \mathrm{y} 7$ & 697.8 & 836.5 & 701.9 & 844.5 & 34.0 \\
\hline & & $+2 \mathrm{y} 6$ & 697.8 & 737.4 & 701.9 & 745.4 & 34.0 \\
\hline \multirow{3}{*}{ LGALS3BP } & \multirow{3}{*}{ YSSDYFQAPSDYR * } & $+2 \mathrm{y}^{9}$ & 799.8 & 1146.5 & 804.8 & 1156.5 & 37.7 \\
\hline & & $+2 \mathrm{y} 8$ & 799.8 & 983.5 & 804.8 & 993.5 & 37.7 \\
\hline & & $+2 y 7$ & 799.8 & 836.4 & 804.8 & 846.4 & 37.7 \\
\hline
\end{tabular}

${ }^{*}$ represents an amino acid labeled with a heavy isotope, ${ }^{13} \mathrm{C}^{15} \mathrm{~N}$. Precursor/fragment ions in bold are used for quantification. The transitions (Q1/Q3) were optimized using Skyline software. Abbreviations: ABL, serum albumin; THBS 1, thrombospondin 1; A2M, alpha-2-macroglobulin; LGALS3BP, galectin 3 binding protein; CE, collision energy.

A

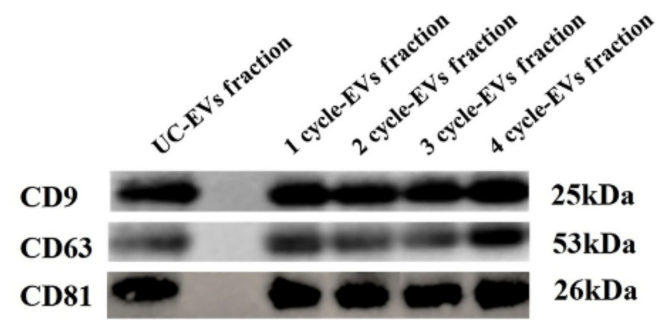

B

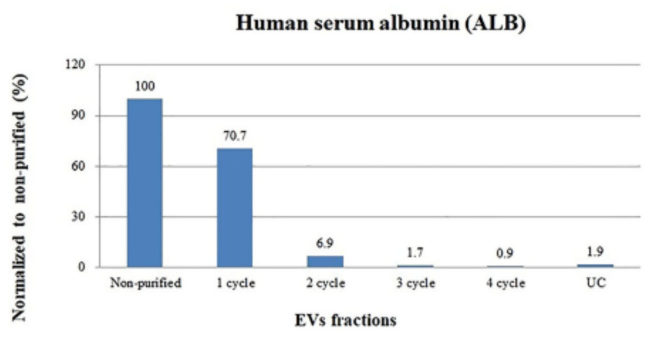

C
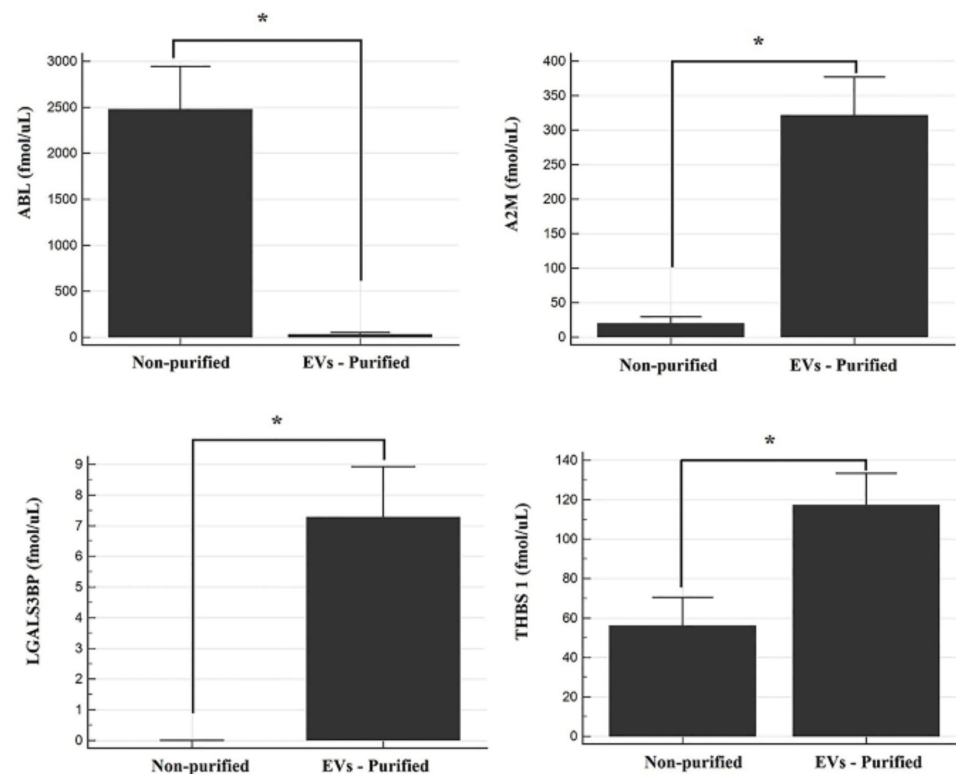

Figure 3. The purity evaluation. (A) Western blot analysis of CD9, CD63, and CD81 on 1-4-cycle EV fractions. (B) The ALB levels measured in EV fractions (1-4 cycles) and UC using an MRM assay. (C) The levels of 3 EV markers (A2M, LGALS3BP, and THBS 1) and ALB in non-purified and EV-purified fractions (2-cycle) obtained by an MRM assay. ${ }^{*}, p$-value $<0.0001$.

Serum albumin (ALB) was used to assess whether serum-derived contaminant proteins were removed or not because it is the most abundant component in the blood sample. We measured the ALB concentrations in the 1-4-cycle EV fractions and UC fraction obtained from the pooled sera. One treatment of polymeric reagents was insufficient for the removal of serum-derived contaminants. Approximately $30 \%$ of ALB was removed in the one-cycle EV fraction, $93 \%$ after two cycles, $99 \%$ with four cycles, and $98 \%$ for the UC fraction (four-cycle) compared with the non-purified fraction (Figure 3B). To verify the above results, we performed an MRM assay of ALB and three EV proteins of the two-cycle 
EV fractions and non-purified fractions obtained from 20 serum samples (Figure 3C). Consistent with previous MRM results, these proteins (A2M, THBS1, and LGALS3BP) increased significantly after EV purification, while the ALB levels were dramatically reduced up to $99 \%$ after EV purification ( $p$-value $<0.0001$ ). Interestingly, LGALS3BP was not detectable in the non-purified fraction but was 7.3-fold enriched after EV purification.

As a result, our data demonstrate that multiple-cycle polymeric EV precipitation is excellent at removing serum-derived contaminant proteins and enriching EV-related proteins. Therefore, we expect the MRM panel consisting of three EV proteins and ALB to be useful for assessing EV purity isolated by various techniques.

\section{Experimental Section}

\subsection{Clinical Samples}

This study was approved by the Institutional Review Board (IRB) of Samsung Medical Center (Seoul, Korea) (IRB file No. 2008-06-007-026). We recruited 10 healthy controls (HC) and 10 patients with pancreatic cancer (PC) for EV purity assessment and LC-MS/MS EV fraction experiments. All serum specimens were obtained by centrifugation of blood at $2330 \times g$ for $5 \mathrm{~min}$ and stored at $-70{ }^{\circ} \mathrm{C}$ for further analysis.

\subsection{EV Purification}

The EVs isolation and characterization were performed according to the exosome guidelines [24]. EV fractions were acquired from UC and a multiple-cycle polymeric EV precipitation method. For the UC fractions, $4 \mathrm{~mL}$ of pooled human serum was centrifuged at $10,000 \times g$ for $30 \mathrm{~min}$, and the supernatant was filtered through a $0.2 \mu \mathrm{m}$ PVDF filter (Agilent Technologies, Santa Clara, CA, USA). The samples were mixed with $6 \mathrm{~mL}$ of PBS and then ultracentrifuged at $100,000 \times g$ for $120 \mathrm{~min}$ at $4{ }^{\circ} \mathrm{C}$ (four times). After the UC step, the supernatant was suctioned, and pellets were reconstituted with $100 \mu \mathrm{L}$ of PBS.

For polymeric EV precipitation, ExoQuick reagent (System Biosciences Inc., Palo Alto, CA, USA) was used according to the manufacturer's recommendations. Briefly, the collected serum was centrifuged at $3000 \times g$ for $15 \mathrm{~min}$ to remove cellular debris. We mixed $25 \mu \mathrm{L}$ of ExoQuick reagent and $100 \mu \mathrm{L}$ of the supernatant were mixed and incubated for $30 \mathrm{~min}$, followed by centrifugation at $1500 \times \mathrm{g}$ for $30 \mathrm{~min}$ at $4{ }^{\circ} \mathrm{C}$. The supernatant was discarded, and the remaining material was centrifuged once more $(1500 \times g$ for $5 \mathrm{~min})$ to remove residual fluid. This step was repeated up to 4 times. The final pellets were reconstituted in $100 \mu \mathrm{L}$ of PBS (Figure 4).

\subsection{Dynamic Light Scattering (DLS) Technology}

DLS measurements were performed using a Zetasizer Nano ZS (Malvern Instruments, Malvern, UK) to determine the particle size distribution of the EVs isolated from different methods. The isolated EVs were diluted 1000-fold with deionized water and tested using a Zetasizer Nano ZS according to the manufacturer's instructions.

\subsection{Transmission Electron Microscopy (TEM)}

We fixed $5 \mu \mathrm{L}$ of freshly prepared EVs with $5 \mu \mathrm{L}$ of $4 \%$ paraformaldehyde for $5 \mathrm{~min}$ at room temperature. About $10 \mu \mathrm{L}$ of the mixture was dropped onto carbon-coated copper grids and then air-dried for $40 \mathrm{~min}$. After washing with PBS, the sample was fixed with $2.5 \%$ glutaraldehyde for $5 \mathrm{~min}$ and stained with UranyLess (Electron Microscopy Sciences, Hatfield, PA, USA) for $2 \mathrm{~min}$, followed by washing in distilled water. The sample was dried and subjected to transmission electron microscopy (TEM) analysis. 


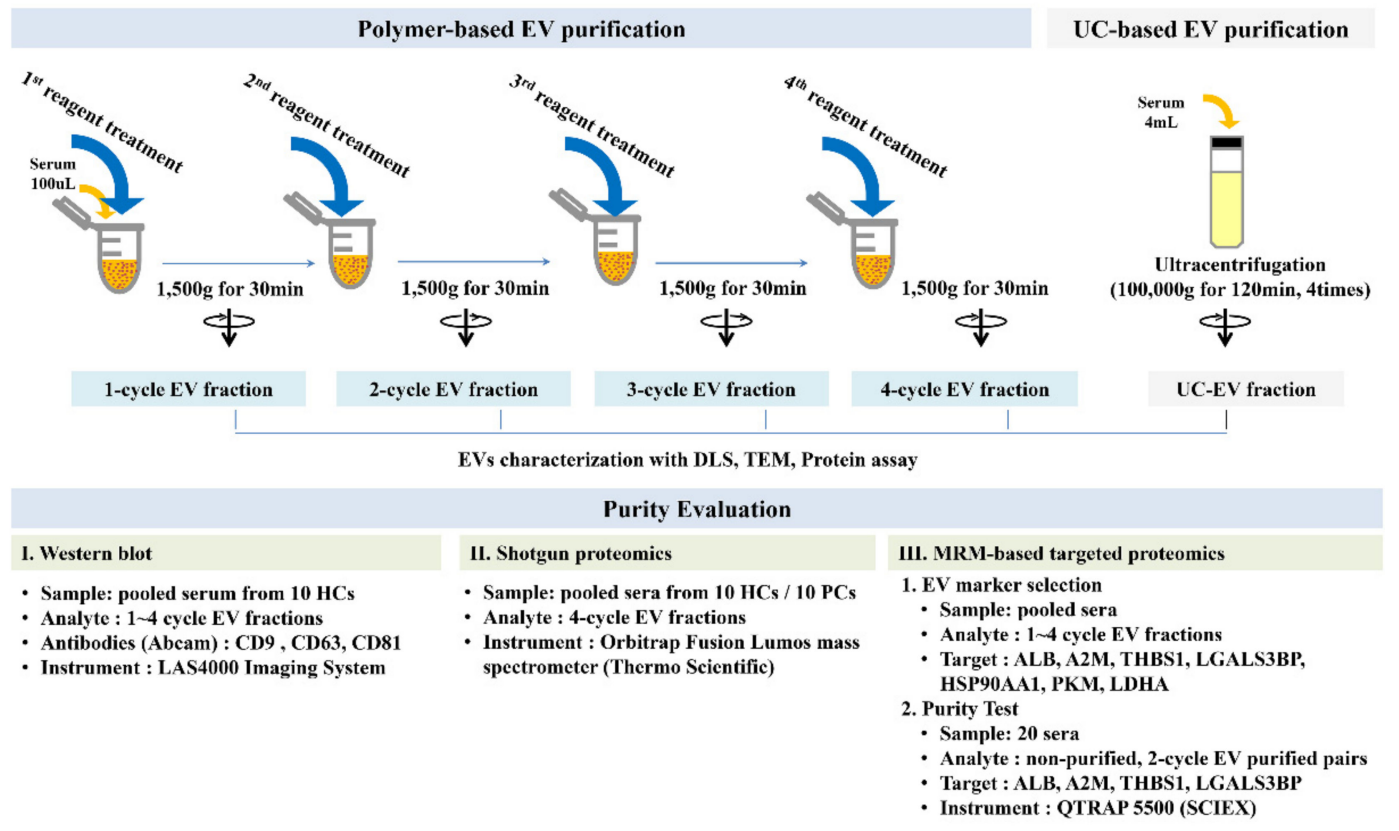

Figure 4. Experimental summary. The EV fraction was obtained by 1 to 4 cycles of polymeric reagent treatment and UC-EV after four repeated cycles of ultracentrifugation. DLS, TEM, and protein assays were performed on these EV fractions. EV proteins candidates were assessed in the 1-4-cycle EV fractions for MRM panel development for the purity test. For the purity test, we performed 2- and 4-cycle reagent treatment for MRM-based targeted proteomics analysis and shotgun proteomic analysis, respectively.

\subsection{Western Blot Analysis}

The protein lysate of the isolated EVs was separated on 4-12\% NuPAGEL Bis-Tris gel (Invitrogen, Carlsbad, CA, USA) and transferred to an Immun-Blot PVDF membrane (BioRad, Hercules, CA, USA). The membranes were blocked in 3\% BSA in phosphate-buffered saline (PBS) overnight at $4{ }^{\circ} \mathrm{C}$ and then incubated with primary antibodies: anti-CD9 (1:1000; Abcam, Cambridge, UK), anti-CD63 (1:1000; Abcam, Cambridge, UK), and antiCD81 (1:1000; Abcam, Cambridge, UK) for $2 \mathrm{~h}$ at room temperature. The membranes were washed three times with PBS, incubated with the corresponding secondary antibody, anti-rabbit (1:3000), for $1 \mathrm{~h}$ at room temperature, and washed with PBST. Signals were visualized after incubation using a Pierce ECL (Thermo, Rockford, IL, USA) and a LAS4000 Imaging System (Fujifilm Life Science, Tokyo, Japan).

\subsection{Shotgun Proteomics Analysis}

A total $500 \mu \mathrm{g}$ of purified EV fractions obtained from pooled sera were subjected to in-solution digestion with trypsin followed by nano-LC-MS/MS analysis. The peptide mixtures were fractionated by reverse phase HPLC $(4.6 \times 150 \mathrm{~mm}$ ID, $2.5 \mu \mathrm{m}$ particle $)$ into 42 fractions. Then, the 42 fractions were combined into 10 fractions and analyzed using an Orbitrap Fusion Lumos mass spectrometer (Thermo Finnigan, San Jose, CA, USA) equipped with a nano-electrospray ion source.

The peptide mixtures were loaded onto a trap column (Thermo Scientific EASYSpray LC Columns $160 \mathrm{~nL}$ enrichment column and $75 \mu \mathrm{m} \times 50 \mathrm{~cm}$ packed C18, $2 \mu \mathrm{m}$ particles, 100 A) connected to the Orbitrap Fusion Lumos mass spectrometer at a flow rate of $4 \mu \mathrm{L} / \mathrm{min}$. The mobile phase consisted of buffer A $(0.1 \%$ formic acid in water) and buffer $\mathrm{B}(0.1 \%$ formic acid in ACN). After injecting a sample onto the column, a 120 min gradient method was used to separate the peptide mixture. Sample loading onto the analytical column was performed at $3 \%$ buffer B, the mobile phase was held at $4 \%$ buffer B for $1 \mathrm{~min}$, followed by a linear gradient to $32 \%$ buffer B over $91 \mathrm{~min}$ and then a linear gradient to $80 \%$ buffer $\mathrm{B}$ over $8 \mathrm{~min}$ at a flow rate of $300 \mathrm{~nL} / \mathrm{min}$. 
All MS/MS spectra were searched against Uniprot Human 2018.1.24 using the ProLuCID search algorithm [25] for peptide identification. The search parameters were as follows: specific to trypsin with two missed cleavages, variable modification of methionine oxidation, fixed modification of carbamidomethyl cysteine, $\pm 10 \mathrm{ppm}$ precursor-ion tolerance, $\pm 600 \mathrm{ppm}$ fragment-ion tolerance, and \pm 10 reporter ion tolerance.

\subsection{MRM-Based Targeted Proteomics Analysis for Purity}

To select EV-specific marker proteins, we used our shotgun data and a list of the top $100 \mathrm{EV}$ proteins released in the ExoCarta depository (http:/ / exocarta.org/index.html, accessed on 10 April 2019) that were frequently detected as a result of various EV analyses including mass spectrometry. First, the top $100 \mathrm{EV}$ proteins were compared with our proteome shotgun data representing evidence of expression in the EV fractions. Of them, the proteins common to both datasets were chosen. Second, the MRM methods were generated using an in-silico approach using Skyline 20.2 (MacCoss Lab Software, Seattle, WA, USA) [26]; upon further refinement of the selected peptides, the transitions were employed. At least three transitions from one proteotypic peptide were generated with 2 or 3 peptide charge states containing 8-30 amino acids. In addition, no post-translational modification (PTM) was applied, and non-specific cleavages were not allowed. Third, the MRM assays were experimentally refined using the EV fractions obtained from the pooled serum sample. Fourth, we determined the proteins selected in the third step using stable isotope dilution (SID)-MRM in non-purified and EV fractions. Then, we selected proteins that increased in the EV-purified fractions for purity evaluation.

We achieved MRM using a QTRAP 5500 hybrid triple quadrupole/linear ion trap mass spectrometer (Sciex, Framingham, MA, USA) equipped with an electrospray ion source. An MRM scan was performed in positive mode with an ion spray voltage of $5500 \mathrm{~V}$. The MRM mode setting was as follows: curtain gas and spray gas were 30 and 50, respectively, and the collision gas was set to the medium level. The declustering potential (DP) was set to $57 \mathrm{~V}$. The mass resolution was set to the units of the advanced MS parameter. The tryptic peptides were loaded into an InfinityLab poroshell 120 EC-C18 (Agilent, $2.1 \times 50 \mathrm{~mm}$ ) packed with C18 $(2.7 \mu \mathrm{m}, 120 \AA)$ reverse-phase resin and separated by LC using a linear gradient of $5-40 \%$ buffer B for 6 min followed by $40-95 \%$ buffer B over 4 min at a flow rate of $0.4 \mathrm{~mL} / \mathrm{min}$.

\subsection{Statistical Analysis}

The acquired MRM assays were analyzed using MedCalc software version 19.0.7 (Mariakerke, Belgium). Differences in protein concentrations between purified and nonpurified EV fractions were assessed by the nonparametric Wilcoxon test; $p \leq 0.05$ was considered statistically significant.

\section{Conclusions}

This is the first report highlighting the usefulness of multiple-cycle polymeric EV precipitation and an MRM panel for purity evaluation. Here, we demonstrated the usefulness of this method through EV shotgun proteomics and EV protein quantification using an MRM assay. In particular, we demonstrated that a single polymeric reagent treatment is vulnerable to serum-derived contamination and does not provide sufficiently high purity for proteomics studies, based on our MRM assay results.

We were able to identify more EV-related proteins including plasma membrane proteins via multiple-cycle polymeric EV precipitation than those obtained using the conventional UC method. Even though further validation experiments are necessary in a large-batch clinical sample, our multiple-cycle polymer-based EV purification method with a high recovery rate using a small sample volume is expected to facilitate various clinical applications of EVs. 
Supplementary Materials: The following are available online at https://www.mdpi.com/article/10 $.3390 /$ ijms22094311/s1.

Author Contributions: Conceptualization, J.P. and S.-Y.L.; methodology, J.P., E.-B.G., and J.S.O.; formal analysis; J.P.; investigation, E.-B.G. and J.S.O.; data curation, J.P. and E.-B.G.; writing-original draft preparation, J.P. and J.S.O.; resources, J.K.L.; writing-review and editing, J.K.L. and S.-Y.L.; project administration, S.-Y.L.; funding acquisition, S.-Y.L. All authors have read and agreed to the published version of the manuscript.

Funding: This work was supported by a National Research Foundation of Korea (NRF) grant funded by the Korea government (MSIT) (No. NRF-2020R1I1A1A01057604, NRF-2021R1A2C1006409).

Institutional Review Board Statement: This study was approved by the Institutional Review Board (IRB) of Samsung Medical Center (Seoul, Korea) (IRB file No. 2008-06-007-026).

Informed Consent Statement: Informed consent was obtained from all patients involved in the study.

Data Availability Statement: The data presented in this study are available in the article or Supplementary Materials.

Conflicts of Interest: The authors declare no conflict of interest.

$\begin{array}{ll}\text { Abbreviations } & \\ \text { EV } & \text { Extracellular vesicle } \\ \text { UC } & \text { Ultracentrifugation } \\ \text { MRM } & \text { Multiple reaction monitoring } \\ \text { A2M } & \text { Alpha-2-microglobulin } \\ \text { THBS 1 } & \text { Thrombospondin 1 } \\ \text { LGALS3BP } & \text { Galectin 3 binding protein } \\ \text { ALB } & \text { Serum albumin } \\ \text { HC } & \text { Healthy control } \\ \text { PC } & \text { Pancreatic cancer } \\ \text { DLS } & \text { Dynamic light scattering } \\ \text { TEM } & \text { Transmission electron microscopy } \\ \text { PBS } & \text { Phosphate-buffered saline } \\ \text { PTM } & \text { Post translational modification } \\ \text { SID } & \text { Stable isotope dilution } \\ \text { DP } & \text { Declustering potential } \\ \text { CD } & \text { Cluster of differentiation }\end{array}$

\section{References}

1. Gyorgy, B.; Szabo, T.G.; Pasztoi, M.; Pal, Z.; Misjak, P.; Aradi, B.; Laszlo, V.; Pallinger, E.; Pap, E.; Kittel, A.; et al. Membrane vesicles, current state-of-the-art: Emerging role of extracellular vesicles. Cell. Mol. Life Sci. 2011, 68, 2667-2688. [CrossRef] [PubMed]

2. Stoorvogel, W.; Kleijmeer, M.J.; Geuze, H.J.; Raposo, G. The biogenesis and functions of exosomes. Traffic 2002, 3, 321-330. [CrossRef]

3. Raposo, G.; Stoorvogel, W. Extracellular vesicles: Exosomes, microvesicles, and friends. J. Cell Biol. 2013, 200, 373-383. [CrossRef] [PubMed]

4. Yanez-Mo, M.; Siljander, P.R.; Andreu, Z.; Zavec, A.B.; Borras, F.E.; Buzas, E.I.; Buzas, K.; Casal, E.; Cappello, F.; Carvalho, J.; et al. Biological properties of extracellular vesicles and their physiological functions. J. Extracell. Vesicles 2015, 4, 27066. [CrossRef] [PubMed]

5. Zoller, M. Exosomes in Cancer Disease. Methods Mol. Biol. 2016, 1381, 111-149. [PubMed]

6. Robbins, P.D.; Morelli, A.E. Regulation of immune responses by extracellular vesicles. Nat. Rev. Immunol. 2014, 14, 195-208. [CrossRef]

7. Bang, C.; Thum, T. Exosomes: New players in cell-cell communication. Int. J. Biochem. Cell. Biol. 2012, 44, 2060-2064. [CrossRef]

8. Choi, D.S.; Kim, D.K.; Kim, Y.K.; Gho, Y.S. Proteomics of extracellular vesicles: Exosomes and ectosomes. Mass Spectrom. Rev. 2015, 34, 474-490. [CrossRef]

9. Fujita, Y.; Yoshioka, Y.; Ochiya, T. Extracellular vesicle transfer of cancer pathogenic components. Cancer Sci. 2016, 107, 385-390. [CrossRef]

10. Properzi, F.; Logozzi, M.; Fais, S. Exosomes: The future of biomarkers in medicine. Biomark. Med. 2013, 7, 769-778. [CrossRef] 
11. El Andaloussi, S.; Mager, I.; Breakefield, X.O.; Wood, M.J. Extracellular vesicles: Biology and emerging therapeutic opportunities. Nat. Rev. Drug Discov. 2013, 12, 347-357. [CrossRef] [PubMed]

12. Rodrigues, M.L.; Nakayasu, E.S.; Almeida, I.C.; Nimrichter, L. The impact of proteomics on the understanding of functions and biogenesis of fungal extracellular vesicles. J. Proteom. 2014, 97, 177-186. [CrossRef] [PubMed]

13. Raimondo, F.; Morosi, L.; Chinello, C.; Magni, F.; Pitto, M. Advances in membranous vesicle and exosome proteomics improving biological understanding and biomarker discovery. Proteomics 2011, 11, 709-720. [CrossRef] [PubMed]

14. Simpson, R.J.; Lim, J.W.; Moritz, R.L.; Mathivanan, S. Exosomes: Proteomic insights and diagnostic potential. Expert Rev. Proteom. 2009, 6, 267-283. [CrossRef]

15. Gonzales, P.A.; Pisitkun, T.; Hoffert, J.D.; Tchapyjnikov, D.; Star, R.A.; Kleta, R.; Wang, N.S.; Knepper, M.A. Large-scale proteomics and phosphoproteomics of urinary exosomes. J. Am. Soc. Nephrol. 2009, 20, 363-379. [CrossRef]

16. Li, Y.; Zhang, Y.; Qiu, F.; Qiu, Z. Proteomic identification of exosomal LRG1: A potential urinary biomarker for detecting NSCLC. Electrophoresis 2011, 32, 1976-1983. [CrossRef] [PubMed]

17. Thery, C.; Amigorena, S.; Raposo, G.; Clayton, A. Isolation and characterization of exosomes from cell culture supernatants and biological fluids. Curr. Protoc. Cell Biol. 2006, 30, 3-22. [CrossRef]

18. Helwa, I.; Cai, J.; Drewry, M.D.; Zimmerman, A.; Dinkins, M.B.; Khaled, M.L.; Seremwe, M.; Dismuke, W.M.; Bieberich, E.; Stamer W.D.; et al. A Comparative Study of Serum Exosome Isolation Using Differential Ultracentrifugation and Three Commercial Reagents. PLOS ONE 2017, 12, e0170628. [CrossRef]

19. Li, P.; Kaslan, M.; Lee, S.H.; Yao, J.; Gao, Z. Progress in Exosome Isolation Techniques. Theranostics 2017, 7, 789-804. [CrossRef]

20. Abramowicz, A.; Widlak, P.; Pietrowska, M. Proteomic analysis of exosomal cargo: The challenge of high purity vesicle isolation. Mol. Biosyst. 2016, 12, 1407-1419. [CrossRef]

21. Kim, J.; Tan, Z.; Lubman, D.M. Exosome enrichment of human serum using multiple cycles of centrifugation. Electrophoresis 2015 36, 2017-2026. [CrossRef] [PubMed]

22. Caradec, J.; Kharmate, G.; Hosseini-Beheshti, E.; Adomat, H.; Gleave, M.; Guns, E. Reproducibility and efficiency of serum-derived exosome extraction methods. Clin. Biochem. 2014, 47, 1286-1292. [CrossRef] [PubMed]

23. Witwer, K.W.; Buzas, E.I.; Bemis, L.T.; Bora, A.; Lasser, C.; Lotvall, J.; Nolte-'t Hoen, E.N.; Piper, M.G.; Sivaraman, S.; Skog, J.; et al. Standardization of sample collection, isolation and analysis methods in extracellular vesicle research. J. Extracell. Vesicles 2013, 2. [CrossRef]

24. Thery, C.; Witwer, K.W.; Aikawa, E.; Alcaraz, M.J.; Anderson, J.D.; Andriantsitohaina, R.; Antoniou, A.; Arab, T.; Archer, F.; Atkin-Smith, G.K.; et al. Minimal information for studies of extracellular vesicles 2018 (MISEV2018): A position statement of the International Society for Extracellular Vesicles and update of the MISEV2014 guidelines. J. Extracell Vesicles 2018, 7, 1535750. [CrossRef] [PubMed]

25. Xu, T.; Park, S.K.; Venable, J.D.; Wohlschlegel, J.A.; Diedrich, J.K.; Cociorva, D.; Lu, B.; Liao, L.; Hewel, J.; Han, X.; et al. ProLuCID: An improved SEQUEST-like algorithm with enhanced sensitivity and specificity. J. Proteom. 2015, 129, 16-24. [CrossRef]

26. MacLean, B.; Tomazela, D.M.; Shulman, N.; Chambers, M.; Finney, G.L.; Frewen, B.; Kern, R.; Tabb, D.L.; Liebler, D.C.; MacCoss, M.J. Skyline: An open source document editor for creating and analyzing targeted proteomics experiments. Bioinformatics 2010, 26, 966-968. [CrossRef] 